\title{
DIFFERENTIATION BY FOURIER TRANSFORMATION AND ITS CONNECTION WITH DIFFERENTIATION BY FINITE DIFFERENCING*
}

\author{
BY \\ BEHROOZ COMPANI - TABRIZI AND RICHARD G. GEYER \\ Marathon Oil Company, Denver Research Center, Littleton, Colorado
}

\begin{abstract}
The relation between central-, forward-, and backward-finite differencing and differentiation by Fourier transformation is developed. The conventional rule for differentiation by Fourier transformation of a discretized function, namely, multiplication of the Fourier transform of the function by $i k$ and a subsequent inverse Fourier transformation, is shown to be a first-order approximation to more complete rules. Numerical examples are provided.
\end{abstract}

Introduction. In modeling physical phenomena, it is often necessary to compute the spatial or temporal gradient of the fields or physical property distributions (such as velocity, density, conductivity, etc.). These gradients can be found by different methods, one of which is multiplication of the Fourier transform of the fields by $i k$ and inverse Fourier transformation of the result. Since the boundaries between physical media are often sharp, the physical properties have a corresponding step-like behavior, so that in modeling the physical phenomena one is confronted with the need to take gradients of discretized fields with high-frequency content. In this article it is shown that the $i k$ multiplication rule as applied to discretized functions is a first-order approximation to more complete rules. These rules are derived for differentiation of discretized functions and are related to central-, forward-, backward-finite differencing. It is also shown by examples that while the $i k$ rule fails badly when applied to high-frequency fields, the new rules give the exact finite-difference results.

Central-finite differencing relation. Consider a function $f(x)$, discretized at $N$ points from $x=-N / 2$ to $x=N / 2$ in equal intervals of $\Delta x$. To perform a Fourier transformation on this function spatial and spectral sampling is chosen as

$$
\begin{array}{ll}
x=\mu \Delta x, & \mu=-N / 2,-N / 2+1, \ldots, 0,1,2, \ldots, N / 2, \\
k=\nu \Delta k, & \nu=-N / 2,-N / 2+1, \ldots, 0,1,2, \ldots, N / 2,
\end{array}
$$

\footnotetext{
* Received June 7, 1985.
} 
where the spatial and spectral discretizations are related by

$$
\Delta x \Delta k=2 \pi / N
$$

which is the minimal relationship imposed on the uncertainties in any two dual spaces by the connection between their Fourier transforms.

It is now shown that central-finite differencing will lead to a relation for differentiation by Fourier transformation that produces exactly the same derivatives as central differencing when used on discretized functions. The examples shown illustrate that the new differentiation rule produces finite-difference derivatives of discretized continuous and discontinuous functions as well as discretized distributions.

Since $\Delta x=2 \pi / N \Delta k$, the central-difference formula for the derivative of $f(x)$ is written as

$$
\frac{d f(x)}{d x}=\frac{f(x+2 \pi / N \Delta k)-f(x-2 \pi / N \Delta k)}{2(2 \pi / N \Delta k)} .
$$

Denoting the convolution operation by * and recognizing that

$$
f\left(x-\frac{2 \pi}{N \Delta k}\right)=\int_{-\infty}^{\infty} \delta\left(x-\frac{2 \pi}{N \Delta k}-x_{0}\right) f\left(x_{0}\right) d x_{0}=\delta\left(x-\frac{2 \pi}{N \Delta k}\right) * f(x),
$$

and

$$
f\left(x+\frac{2 \pi}{N \Delta k}\right)=\int_{-\infty}^{\infty} \delta\left(x+\frac{2 \pi}{N \Delta k}-x_{0}\right) f\left(x_{0}\right) d x_{0}=\delta\left(x+\frac{2 \pi}{N \Delta k}\right) * f(x),
$$

Eq. (4) is written as

$$
\begin{aligned}
\frac{d f(x)}{d x} & =\frac{N \Delta k}{4 \pi}\left[\delta\left(x+\frac{2 \pi}{N \Delta k}\right)-\delta\left(x-\frac{2 \pi}{N \Delta k}\right)\right] * f(x) \\
& =F^{-1} F \frac{N \Delta k}{4 \pi}\left\{\left[\delta\left(x+\frac{2 \pi}{N \Delta k}\right)-\delta\left(x-\frac{2 \pi}{N \Delta k}\right)\right] * f(x)\right\} \\
& =F^{-1} \frac{N \Delta k}{4 \pi} F\left\{\left[\delta\left(x+\frac{2 \pi}{N \Delta k}\right)-\delta\left(x-\frac{2 \pi}{N \Delta k}\right)\right] * f(x)\right\},
\end{aligned}
$$

where $F$ and $F^{-1}$ represent the direct and inverse Fourier transform operations.

Since $\delta(x \pm 2 \pi / N \Delta k)$ and $\exp ( \pm i 2 \pi / N \Delta k)$ are Fourier pairs, and Fourier transformation of the convolution of two functions in the spatial domain leads to multiplication of their Fourier transforms in the spectral domain, Eq. (5) may be written as

$$
\begin{aligned}
\frac{d f(x)}{d x} & =F^{-1}\left\{\frac{N \Delta k}{4 \pi}\left[\exp \left(i \frac{2 \pi}{N \Delta h} k\right)-\exp \left(-i \frac{2 \pi}{N \Delta h} k\right)\right] \cdot F[f(x)]\right\} \\
& =F^{-1}\left\{\frac{i N \Delta k}{2 \pi}\left[\frac{\exp \left(i \frac{2 \pi}{N \Delta h} k\right)-\exp \left(-i \frac{2 \pi}{N \Delta h} k\right)}{2 i}\right] \cdot F[f(x)]\right\}
\end{aligned}
$$


Since $\Delta x=2 \pi / N \Delta k$ and $k=\nu \Delta k$, the following global differentiation formula results:

$$
\begin{aligned}
\frac{d f(x)}{d x} & =\frac{1}{\Delta x} F^{-1}\{i \sin (2 \pi k / N \Delta k) \cdot F[f(x)]\} \\
& =\frac{1}{\Delta x} F^{-1}\{i \sin (2 \pi \nu / N) \cdot F[f(x)]\} .
\end{aligned}
$$

Note that since

$$
\sin \left(\frac{2 \pi}{N \Delta k} k\right)=\left(\frac{2 \pi}{N \Delta k} k\right)-\frac{1}{3 !}\left(\frac{2 \pi}{N \Delta k} k\right)^{3}+\frac{1}{5 !}\left(\frac{2 \pi}{N \Delta k} k\right)^{5} \cdots,
$$

Equation (7) can be expressed as

$$
\begin{aligned}
\frac{d f(x)}{d x} & =\frac{N \Delta k}{2 \pi} F^{-1}\left\{\left[i \frac{2 \pi}{N \Delta k} k-\frac{i}{3 !}\left(\frac{2 \pi}{N \Delta k} k\right)^{3}+\cdots\right] \cdot F[f(x)]\right\} \\
& =\frac{1}{\Delta x} F^{-1}\left\{\left[i \frac{2 \pi}{N} \nu-\frac{i}{3 !}\left(\frac{2 \pi}{N} \nu\right)^{3}+\cdots\right] \cdot F[f(x)]\right\} .
\end{aligned}
$$

Keeping the first-order term and neglecting higher-order terms, Equation (9) reduces to

$$
\frac{d f(x)}{d x}=F^{-1}\{i k F[f(x)]\}=\frac{1}{\Delta x} F^{-1}\left\{i\left(\frac{2 \pi}{N} \nu\right) F[f(x)]\right\},
$$

which is the usual differentiation rule normally used. However, Equation (10) often does not produce satisfactory results when used on discretized functions, as is later shown in examples.

Generalization of Equation (7) to the $n$th derivative leads to the relation

$$
\frac{d^{n} f(x)}{d x^{n}}=\frac{1}{(\Delta x)^{n}} F^{-1}\left\{\left[i \sin \left(\frac{2 \pi}{N} \nu\right)\right]^{n} \cdot F[f(x)]\right\} \text {. }
$$

Examples of the first, second, and third derivatives are later presented in the form of graphs for distributions and continuous and discontinuous functions.

Forward- and backward-finite differencing relations. The same procedure can be used for finding the Fourier transformation differentiation formula that results from the forwardand backward-finite differencing. The forward relation is now developed.

$$
\begin{aligned}
& \frac{d f(x)}{d x}=\frac{f\left(x+\frac{2 \pi}{N \Delta h}\right)-f(x)}{\frac{2 \pi}{N \Delta h}}=\frac{N \Delta k}{2 \pi}\left[\delta\left(x+\frac{2 \pi}{N \Delta k}\right)-\delta(x)\right] * f(x) \\
& =F^{-1} F \frac{N \Delta k}{2 \pi}\left[\delta\left(x+\frac{2 \pi}{N \Delta k}\right)-\delta(x)\right] * f(x)=F^{-1} \frac{N \Delta k}{2 \pi}\left[\exp \left(i \frac{2 \pi}{N \Delta h} k\right)-1\right] \cdot F[f(x)] \\
& =\frac{1}{\Delta x} F^{-1}\left[\exp \left(i \frac{2 \pi}{N} \nu\right)-1\right] \cdot F[f(x)] \\
& =\frac{1}{\Delta x} F^{-1}\left[i \frac{2 \pi}{N} \nu-\frac{1}{2 !}\left(\frac{2 \pi}{N} \nu\right)^{2}-\frac{i}{3 !}\left(\frac{2 \pi}{N} \nu\right)^{3}+\cdots\right] \cdot F[f(x)] .
\end{aligned}
$$


Similarly, backward differencing leads to the relation

$$
\begin{aligned}
\frac{d f(x)}{d x} & =\frac{1}{\Delta x} F^{-1}\left[1-\exp \left(-i \frac{2 \pi}{N} \nu\right)\right] \cdot F[f(x)] \\
& =\frac{1}{\Delta x} F^{-1}\left[i \frac{2 \pi}{N} \nu+\frac{1}{2 !}\left(\frac{2 \pi}{N} \nu\right)^{2}-\frac{i}{3 !}\left(\frac{2 \pi}{N} \nu\right)^{3}+\cdots\right] \cdot F[f(x)] .
\end{aligned}
$$

Extension of Eqs. (12) and (13) to the $n$th derivative is straightforward.

It is observed that central, forward, and backward differentiation by Fourier transformation relations (i.e., Eqs. (9), (12), and (13)) are exactly the same to the first order in $\nu$. It is also noted that the effect of excluding higher-order terms on differentiation will be felt more strongly in the higher-frequency regions of the function.

Numerical examples. In Figs. 1-3, numerical first, second, and third derivatives of discretized delta, step, and Gaussian functions are exhibited using differentiation by Fourier transformation with both the first order, $2 \pi \nu / N$, as well as the full infinite order

$$
\left.\begin{array}{l}
\text { Imag } \frac{i}{(\Delta x)} F^{-1}\left\{\left(\frac{2 \pi}{N} \nu\right) F[f(x)]\right\} \\
0
\end{array}\right] \text {. }
$$

FIG. 1. First derivative of a "discretized delta" function. Both the real and imaginary parts are shown. Central differencing relations are compared to the conventional rule. 


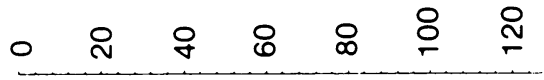

$$
\begin{aligned}
& \text { Imag } \left.\frac{-1}{(\Delta x)^{2}} F^{-1}\left\{\left(\frac{2 \pi}{N} \nu\right) F[f(x)]\right\} \quad \begin{array}{c}
0 \\
-10^{-8}
\end{array}\right] \text {. } \\
& \text { Real } \left.\frac{-1}{(\Delta x)^{2}} F^{-1}\left\{\left(\frac{2 \pi}{N} \nu\right) F[f(x)]\right\} \quad \begin{array}{r}
0 \\
-67
\end{array}\right] \text {. } \\
& \left.\operatorname{Imag} \frac{-1}{(\Delta x)^{2}} F^{-1}\left\{\sin ^{2} \frac{2 \pi}{N} \nu F[f(x)]\right\} \quad \begin{array}{r}
0 \\
-10^{-11}
\end{array}\right] \quad \ldots \ldots \ldots \\
& \text { Real } \frac{-1}{(\Delta x)^{2}} F^{-1}\left\{\sin ^{2} \frac{2 \pi}{N} \nu F[f(x)]\right\} \\
& ]_{-0.5}^{0} \longrightarrow \\
& f(x)= \begin{cases}1 & x=32 \\
0 & x \neq 32\end{cases}
\end{aligned}
$$

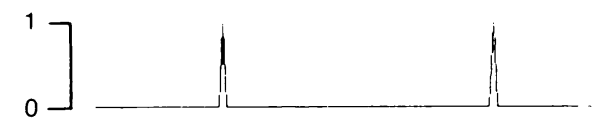

FIG. 2. Second derivative of a "discretized delta" function. Both the real and imaginary parts are shown. Central differencing relations are compared to the conventional rule.

series $\sin (2 \pi \nu / N)$, corresponding to the central differencing differentiation. Figs. 4 and 5 show the differentiation of the same discretized delta function using the forward and backward differentiation relations, respectively. Comparisons show that the oscillations in the derivative resulting from the conventional rule have been corrected, and that the imaginary part of the derivative is negligible in the new formulation, as it should be.

Fig. 6 displays the derivative of a sampled "smooth" Gaussian function using the central difference formulas. For this case, the real part of the conventional Fourier rule and the new Fourier rule results compare. However, the magnitude of the imaginary part of the new Fourier method is smaller than the conventional Fourier method by orders of magnitude. Fig. 7 displays the derivative of a discretized straight line. The conventional method produces a clearly incorrect oscillating derivative. In all cases, 64 points were taken to uniformly sample the function. The Fourier transformation is performed by a fast Fourier transformation algorithm, and the function is made symmetric in the folded region. The plots represent the symmetric function in the full folded domain. In all cases the function and both the real and imaginary parts of the results are presented. 


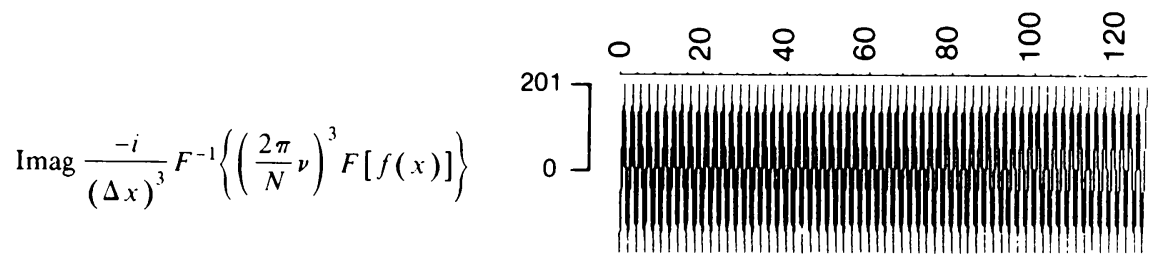

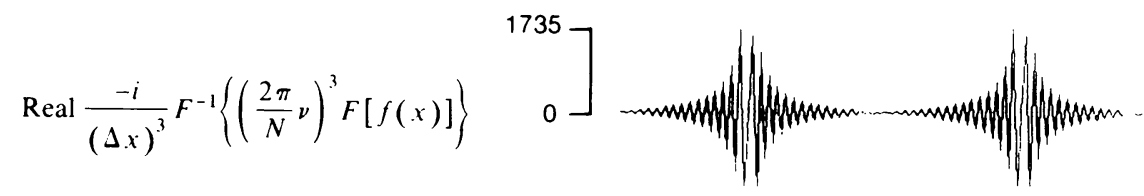

$$
\begin{aligned}
& \operatorname{Imag} \frac{-i}{(\Delta x)^{3}} F^{-1}\left\{\sin ^{3} \frac{2 \pi}{N} \nu F[f(x)]\right\} \\
& \left.\begin{array}{c}
0 \\
-10^{-11}
\end{array}\right] \quad \cdots \\
& \text { Real } \frac{-i}{(\Delta x)^{3}} F^{-1}\left\{\sin ^{3} \frac{2 \pi}{N} \nu F[f(x)]\right\} \\
& 0.375] \\
& f(x)=\left\{\begin{array}{l}
1 x=32 \\
0 x \neq 32
\end{array}\right.
\end{aligned}
$$

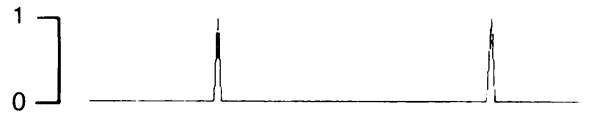

FIG. 3. Third derivative of a "discretized delta" function. Both the real and imaginary parts are shown. Central differencing relations are compared to the conventional rule. 


$$
\operatorname{Imag} \frac{i}{(\Delta x)} F^{-1}\left\{\left(\frac{2 \pi}{N} \nu\right) F[f(x)]\right\}
$$

Real $\frac{i}{(\Delta x)} F^{-1}\left\{\left(\frac{2 \pi}{N} \nu\right) F[f(x)]\right\}$

$\operatorname{Imag} \frac{1}{\Delta x} F^{-1}\left[\exp \left(i_{N}^{2 \pi} \nu\right)-1\right] \cdot F[f(x)]$

$$
\text { Real } \frac{1}{\Delta x} F^{-1}\left[\exp \left(i_{N}^{2 \pi} \nu\right)-1\right] \cdot F[f(x)]
$$

$$
f(x)= \begin{cases}1 & x=32 \\ 0 & x \neq 32\end{cases}
$$
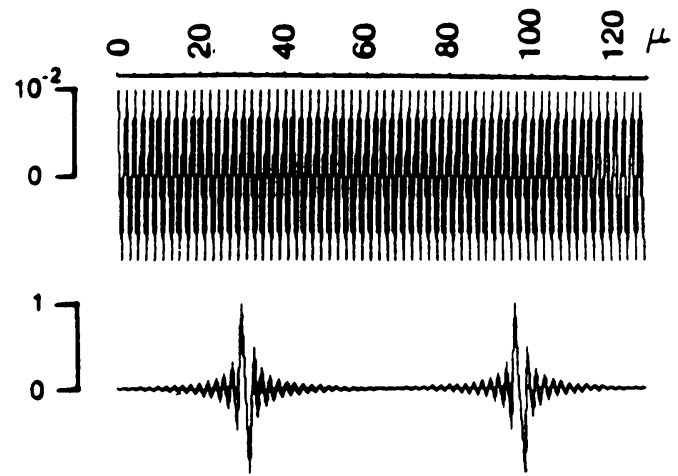

$10^{-10}$
0
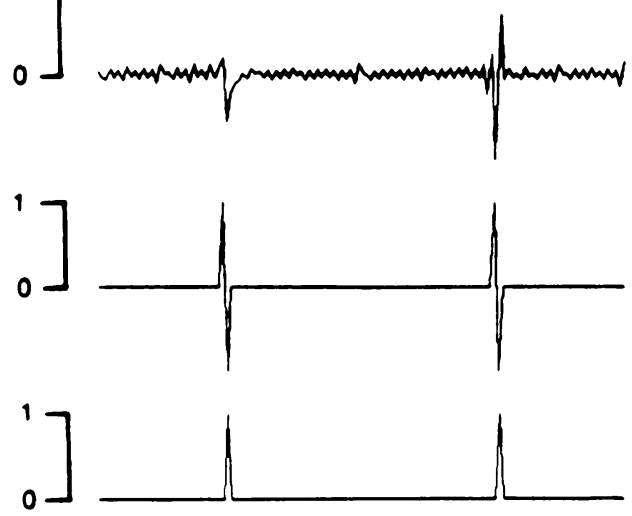

FIG. 4. First derivative of a "discretized delta" function. Both the real and imaginary parts are shown. Forward differencing relations are compared to the conventional rule. 

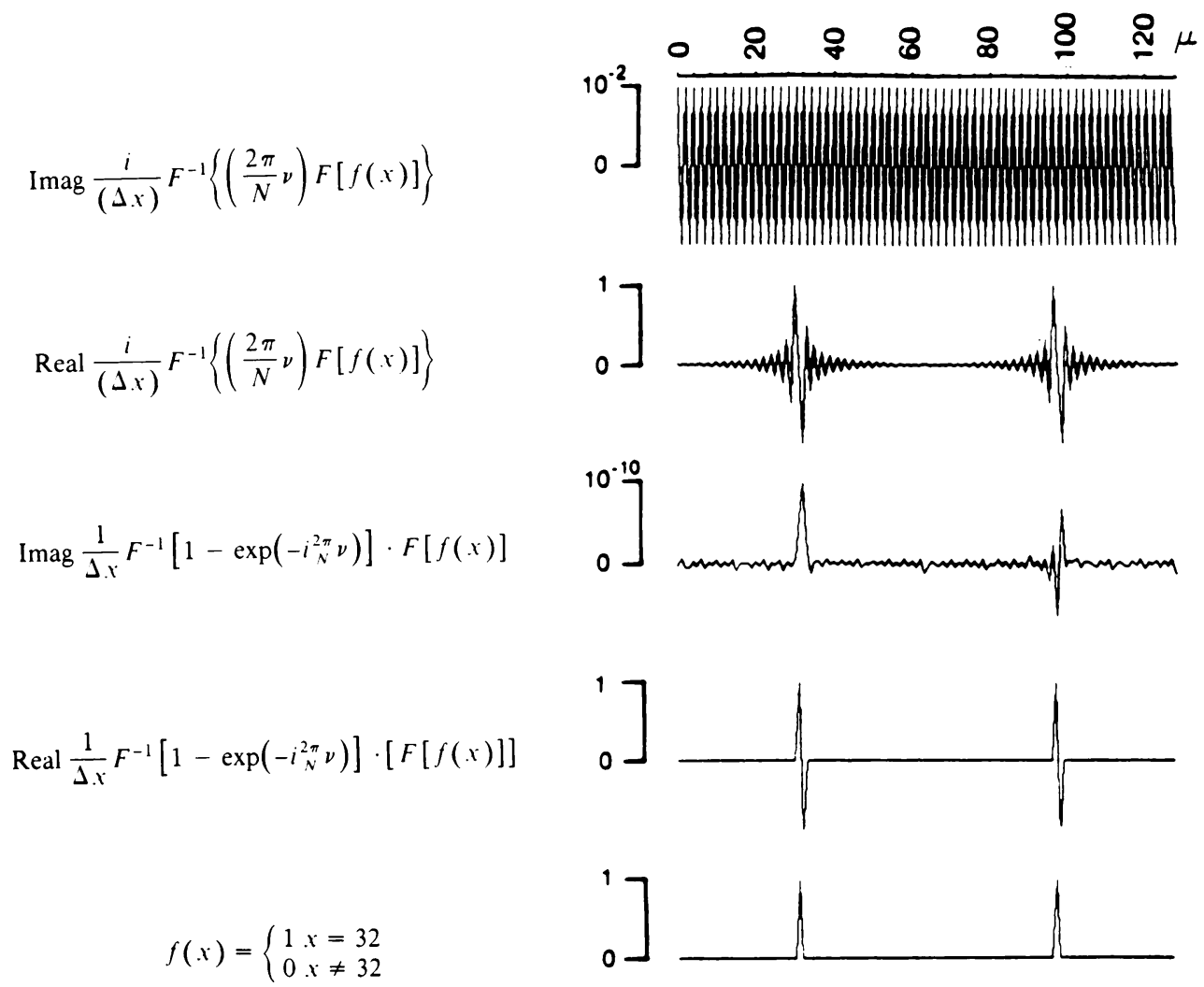

$$
f(x)= \begin{cases}1 & x=32 \\ 0 & x \neq 32\end{cases}
$$

FIG. 5. First derivative of a "discretized delta" function. Both the real and imaginary parts are shown. Backward differencing relations are compared to the conventional rule. 


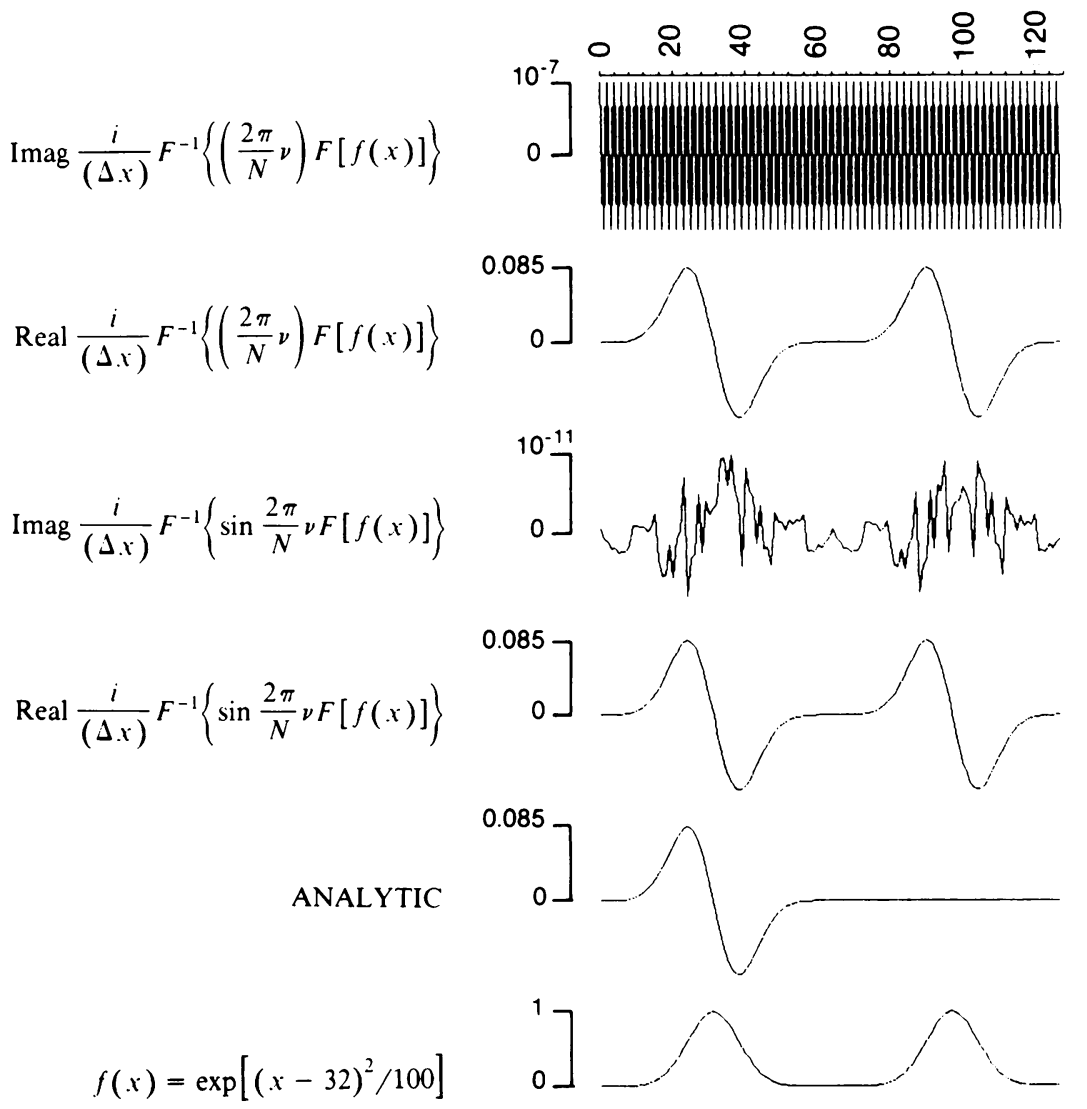

FIG. 6. First derivative of a "smooth function" (Gaussian). Both the real and imaginary parts are shown. Central differencing relations were used and the analytic first derivative is shown. 


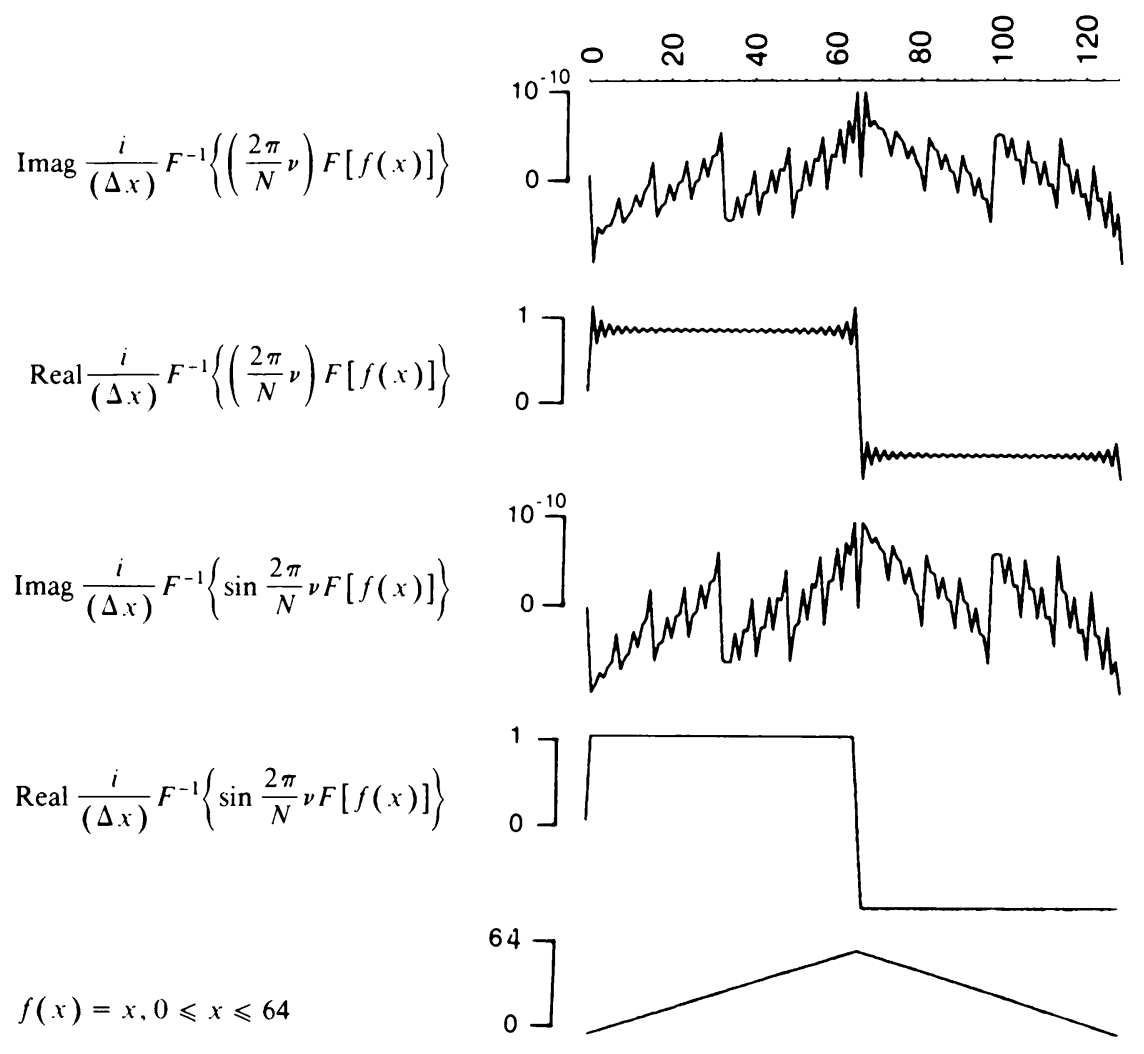

FIG. 7. First derivative of a "smooth function" (straight line). Both the real and imaginary parts are shown. The conventional method produces the derivative with errors up to $40 \%$. 